\title{
Study of Breeding Potential of Brinjal Genotypes (Solanum melongena L.)
}

\author{
Bhagwan Bamaniya*, S. S. Singh and Anita Parmar \\ Department of Crop Sciences, (Mahatma Gandhi Chitrakoot Gramodaya Vishvavidyalaya, \\ Chitrakoot, Satna (MP), India \\ *Corresponding author
}

A B S T R A C T

Keywords

Fruit yield traits, Correlation and path coefficients parameters and grouping various clusters in Brinjal

Article Info

Accepted:

20 December 2020

Available Online:

10 January 2021
A field experiment was conducted during kharif, 2017 and 2018 at Horticulture Research Farm, Mahatma Gandhi Chitrakoot Gramodaya Vishvavidyalaya, Chitrakoot Satna (M.P). Design of experiment was RBD with three replications. Twenty five brinjal genotypes where studied for identification of traits governing fruit yield through correlation and path analysis revealed that days to $50 \%$ flowering fruit span, number of fruits per plant, fruit length, fruit weight and branches per plant found major fruit yield traits. similarly 25 genotypes where grouped in 5 cluster consisting nine cluster II, seven clusters III, nine in cluster I, three in cluster Iv and one in plaster v, finally genotypes namely BCB-464, Utkal Tarahi, KS-331, Aruna, PLR-1 and JB- 69 were found superior for further breeding program.

\section{Introduction}

Brinjal (Solanum melongena L.) belong to family solanaceae is an often cross pollinated crop having cross pollination more than $20 \%$ reported by Madhavi (2015) which caters greater variability traits and diversity in existing genotypes/varieties.

In India it is cultivated in an area of 730 thousand hectares and producing 12801 thousand metric tons with a productivity of 17.5 metric tons per hectare. (Anon, 2018). Regional preferences liking and demand differ greatly with size, shape, colour of fruits and prickles on the calyx attributes. This has created the primary breeding objective to breed new brinjal varieties. This may fulfill the area specific needs of the growers and consumers. Planning and execution of a breeding programme for the improvement of the various quantitative attributes and quantitative depends on the magnitude of genetic variability already present in the base population. Evaluation of extent of variability available for the fruit yield and it's attributes with heritability is of immense importance to the breeders to select highly heritable traits contributed to fruit yield for genetic improvement in yield and quality. Hence an 
attempt has been made to assess the available genetic variability by the partitioning of overall variability into its heritable and non heritable components based on genetic parameters e.g., genotypic and phenotypic coefficient of variation, heritability and expected genetic advance as percentage of mean. Knowledge on nature and magnitude of variation present in available breeding materials are essential pre requisites to choose the characters for effective selection of desirable genotypes to be undertaken for crop improvement programme.

In any crop improvement programme, genetic diversity plays a very important role as it helps in selecting the suitable parents for hybridization programme resulting for breeding superior hybrids with desirable recombinants.

The genetic variability forms the basis of the entire breeding programme selection cannot be effective in population without variability. In terms of variability, it is the genetic fraction of the observed variation that provides a measure of the transmissibility of the variation under study and responds to selection.

These is an almost need for development of high yielding varieties and hybrids for specified area (Vaddoria et al.2009). Brinjal having high variation with regard to fruit yield and its components and genetic divergence based on Mahanobis $\mathrm{D}^{2}$ techniques described by Rao (1952). Appears to be a fruitful method based on multivariate analysis serves a good index of Genetic diversity helps to breed further suitable commercial variety of Brinjal.

Correlation and path Coefficient studies provide information's on degree add direction of fruit yield and its components Sithi Saha et. al. 2019)

\section{Materials and Methods}

The field experiment was carried out at the research field of horticulture form Mahatma Gandhi Chitrakoot gramodaya Vishwavidyalaya Chitrakoot Satna(MP). 25 genotypes/ varieties of brinjal from genetic stock and grown in RBD with three replications.

Before sowing seeds were treatments with Bavistine (Carbandazim 50\% D.F) for 5 minutes and 25 days old seedlings were transplanted from nursery to lots of having many well drained soils. The manures and fertilizers were applied 1.5 ton $/ \mathrm{h}$ and 200:60:100 NPK per hectare.

The first instalments were given after 21 DAT and second were given at 50 DAT. The plots were then irrigated frequently to avoid water stress. During crop period Ripcord 10 EC was spread at 15 days interval to prevent disease occurrence. Data were collected on different Physio morphic growth and fruit yield parameters.

The parameters namely correlation coefficients of fruit yield and its traits were determined as per Singh and Choudhary (1985). Direct and indirect effects of various characters on fruit where calculated, through procedure given by Dewey and Lu (1930) and divergence estimated as per procedure given by Smith (1936) and group constellation carried out as per method proposed by Rao (1952) were used in the present investigation.

\section{Results and Discussion}

25 diverse genotypes of brinjal for 12 characters were studies for identification of major fruits yield traits through correlation and path coefficients parameters and grouping than into various clusters through touches (Rao 1952) method. 
Table.1 Estimates of genotypic and phenotypic correlation coefficients among yield and its attributing traits in brinjal in brinjal in First year

\begin{tabular}{|c|c|c|c|c|c|c|c|c|c|c|c|c|c|c|}
\hline Characters & & $\begin{array}{c}\text { Plant } \\
\text { height } \\
(\mathrm{cm}) \text { at } \\
90 \text { DAT }\end{array}$ & $\begin{array}{l}\text { Leaves/ } \\
\text { plant at } \\
90 \text { DAT }\end{array}$ & $\begin{array}{c}\text { Branches } \\
\text { /plant } \\
\text { at90 } \\
\text { DAT }\end{array}$ & $\begin{array}{c}\text { Days to } \\
\text { first } \\
\text { flowering }\end{array}$ & \begin{tabular}{|c|} 
Days to \\
$50 \%$ \\
flowering
\end{tabular} & $\begin{array}{c}\text { Days to } \\
\text { first } \\
\text { picking }\end{array}$ & $\begin{array}{c}\text { Fruiting } \\
\text { span }\end{array}$ & $\begin{array}{l}\text { No. of } \\
\text { fruits / } \\
\text { plant }\end{array}$ & $\begin{array}{l}\text { Fruit } \\
\text { length } \\
\text { (cm) }\end{array}$ & $\begin{array}{c}\text { Fruit } \\
\text { width } \\
\text { (cm) }\end{array}$ & $\begin{array}{c}\text { Fruit } \\
\text { circumfere } \\
\text { nce }(\mathbf{c m})\end{array}$ & $\begin{array}{l}\text { Weight } \\
\text { of fruit } \\
\text { (g) }\end{array}$ & $\begin{array}{c}\text { Fruit } \\
\text { yield / } \\
\text { plant (g) }\end{array}$ \\
\hline \multirow{2}{*}{$\begin{array}{c}\text { Plant height }(\mathrm{cm}) \\
\text { at90 DAT }\end{array}$} & $\mathrm{G}$ & 1.000 & 0.652 & 0.617 & -0.616 & 0.318 & -0.525 & -0.228 & 0.021 & 0.103 & 0.630 & 0.622 & -0.180 & -0.069 \\
\hline & $\mathrm{P}$ & 1.000 & $0.475 * *$ & $0.452 * *$ & $-0.496^{* *}$ & $0.237 *$ & $-0.375 * *$ & -0.137 & -0.003 & 0.069 & $0.519 * *$ & $0.531 * *$ & -0.135 & -0.040 \\
\hline \multirow{2}{*}{$\begin{array}{c}\text { No. of leaves/ plant } \\
\text { at90 DAT }\end{array}$} & $\mathrm{G}$ & & 1.000 & 0.541 & -0.502 & -0.142 & -0.376 & -0.459 & -0.099 & 0.503 & 0.520 & 0.510 & 0.001 & -0.072 \\
\hline & $\mathrm{P}$ & & 1.000 & $0.399 * *$ & $-0.273 *$ & -0.118 & -0.127 & $-0.331 * *$ & -0.034 & $0.390 * *$ & $0.334 * *$ & $0.347 * *$ & 0.006 & -0.032 \\
\hline \multirow{2}{*}{$\begin{array}{l}\text { No. of branches/ } \\
\text { plant at90 DAT }\end{array}$} & G & & & 1.000 & -0.530 & 0.035 & -0.355 & -0.300 & 0.097 & 0.424 & 0.616 & 0.599 & -0.003 & 0.081 \\
\hline & $\mathrm{P}$ & & & 1.000 & $-0.316^{* *}$ & -0.028 & -0.194 & $-0.260 *$ & 0.132 & $0.303 * *$ & $0.399 * *$ & $0.423 * *$ & -0.003 & 0.075 \\
\hline \multirow{2}{*}{$\begin{array}{l}\text { Days to first } \\
\text { flowering }\end{array}$} & G & & & & 1.000 & 0.023 & 0.294 & -0.167 & -0.192 & -0.228 & -0.117 & -0.119 & 0.083 & -0.155 \\
\hline & $\mathrm{P}$ & & & & 1.000 & 0.089 & 0.160 & -0.100 & -0.178 & -0.124 & -0.050 & -0.046 & 0.032 & -0.081 \\
\hline \multirow{2}{*}{$\begin{array}{c}\text { Days to } 50 \% \\
\text { flowering }\end{array}$} & $\mathrm{G}$ & & & & & 1.000 & -0.064 & -0.035 & 0.371 & -0.109 & 0.181 & 0.164 & 0.214 & 0.376 \\
\hline & $\mathrm{P}$ & & & & & 1.000 & -0.099 & 0.053 & $0.284 *$ & -0.024 & 0.113 & 0.138 & 0.124 & $0.312 * *$ \\
\hline \multirow[t]{2}{*}{ Days to first picking } & G & & & & & & 1.000 & 0.377 & 0.464 & 0.200 & -0.716 & -0.695 & 0.149 & 0.436 \\
\hline & $\mathrm{P}$ & & & & & & 1.000 & $0.253^{*}$ & $0.369 * *$ & 0.161 & $-0.470 * *$ & $-0.498 * *$ & 0.100 & $0.288^{*}$ \\
\hline \multirow[t]{2}{*}{ Fruiting span } & $\mathrm{G}$ & & & & & & & 1.000 & 0.445 & -0.323 & -0.292 & -0.292 & -0.109 & 0.304 \\
\hline & $\mathrm{P}$ & & & & & & & 1.000 & $0.307 * *$ & -0.227 & -0.210 & -0.210 & -0.071 & $0.264^{*}$ \\
\hline \multirow[t]{2}{*}{ No. of fruits / plant } & $\mathrm{G}$ & & & & & & & & 1.000 & 0.095 & -0.195 & -0.194 & 0.298 & 0.944 \\
\hline & $\mathrm{P}$ & & & & & & & & 1.000 & 0.053 & -0.118 & -0.118 & $0.259 *$ & $0.859 * *$ \\
\hline \multirow[t]{2}{*}{ Fruit length $(\mathrm{cm})$} & G & & & & & & & & & 1.000 & -0.242 & -0.241 & 0.372 & 0.202 \\
\hline & $\mathrm{P}$ & & & & & & & & & 1.000 & -0.212 & -0.213 & $0.343 * *$ & 0.183 \\
\hline \multirow[t]{2}{*}{ Fruit width (cm) } & G & & & & & & & & & & 1.000 & 0.915 & -0.199 & -0.222 \\
\hline & $\mathrm{P}$ & & & & & & & & & & 1.000 & $0.886^{* *}$ & -0.146 & -0.125 \\
\hline \multirow{2}{*}{$\begin{array}{l}\text { Fruit circumference } \\
(\mathbf{c m})\end{array}$} & $\mathrm{G}$ & & & & & & & & & & & 1.000 & -0.195 & -0.218 \\
\hline & $\mathrm{P}$ & & & & & & & & & & & 1.000 & -0.152 & -0.130 \\
\hline \multirow[t]{2}{*}{ Weight of fruit (g } & G & & & & & & & & & & & & 1.000 & 0.650 \\
\hline & $\mathrm{P}$ & & & & & & & & & & & & 1.000 & $0.620 * *$ \\
\hline
\end{tabular}


Table.2 Genotypic path coefficients showing direct and indirect effects of different characters on fruit yield /plant (g) in first year

\begin{tabular}{|c|c|c|c|c|c|c|c|c|c|c|c|c|c|}
\hline Characters & $\begin{array}{c}\text { Plant } \\
\text { height } \\
(\mathrm{cm}) \text { at } \\
90 \text { DAT }\end{array}$ & $\begin{array}{l}\text { Leaves/ } \\
\text { plant at } \\
90 \text { DAT }\end{array}$ & $\begin{array}{l}\text { Branches } \\
\text { /plant } \\
\text { at90 DAT }\end{array}$ & $\begin{array}{l}\text { Days to } \\
\text { first } \\
\text { flowering }\end{array}$ & $\begin{array}{c}\text { Days to } \\
50 \% \\
\text { flowering }\end{array}$ & $\begin{array}{l}\text { Days to } \\
\text { first } \\
\text { picking }\end{array}$ & $\begin{array}{c}\text { Fruiting } \\
\text { span }\end{array}$ & $\begin{array}{l}\text { No. of } \\
\text { fruits / } \\
\text { plant }\end{array}$ & $\begin{array}{c}\text { Fruit } \\
\text { length } \\
\text { (cm) }\end{array}$ & $\begin{array}{c}\text { Fruit } \\
\text { width } \\
\text { (cm) }\end{array}$ & $\begin{array}{l}\text { Fruit } \\
\text { circumfe } \\
\text { rence } \\
\text { (cm) }\end{array}$ & $\begin{array}{l}\text { Weight of } \\
\text { fruit (g) }\end{array}$ & $\begin{array}{l}\text { r" value } \\
\text { fruit yield } \\
\text { /plant (g) }\end{array}$ \\
\hline $\begin{array}{c}\text { Plant height }(\mathrm{cm}) \\
\text { at } 90 \text { DAT }\end{array}$ & -0.1637 & 0.1018 & 0.0699 & 0.0486 & 0.0182 & -0.0235 & 0.0115 & 0.0163 & -0.0204 & -0.0391 & -0.0129 & -0.0761 & -0.069 \\
\hline $\begin{array}{c}\text { Leaves/ plant at } 90 \\
\text { DAT }\end{array}$ & -0.1068 & 0.1561 & 0.0612 & 0.0396 & -0.0081 & -0.0168 & 0.0233 & -0.0785 & -0.0994 & -0.0322 & -0.0106 & 0.0004 & -0.072 \\
\hline $\begin{array}{c}\text { Branches/plant } \\
\text { at90 DAT }\end{array}$ & -0.1011 & 0.0844 & 0.1132 & 0.0418 & 0.0020 & -0.0159 & 0.0152 & 0.0770 & -0.0839 & -0.0382 & -0.0125 & -0.0011 & 0.081 \\
\hline $\begin{array}{l}\text { Days to first } \\
\text { flowering }\end{array}$ & 0.1008 & -0.0784 & -0.0600 & -0.0789 & 0.0013 & 0.0131 & 0.0085 & -0.1520 & 0.0451 & 0.0072 & 0.0025 & 0.0353 & -0.155 \\
\hline $\begin{array}{l}\text { Days to } 50 \% \\
\text { flowering }\end{array}$ & -0.0521 & -0.0222 & 0.0040 & -0.0018 & 0.0572 & -0.0029 & 0.0018 & 0.2945 & 0.0216 & -0.0112 & -0.0034 & 0.0907 & 0.376 \\
\hline $\begin{array}{l}\text { Days to first } \\
\text { picking }\end{array}$ & 0.0859 & -0.0587 & -0.0402 & -0.0232 & -0.0037 & 0.0447 & -0.0191 & 0.3685 & -0.0396 & 0.0444 & 0.0145 & 0.0630 & 0.436 \\
\hline Fruiting span & 0.0373 & -0.0717 & -0.0340 & 0.0132 & -0.0020 & 0.0169 & -0.0507 & 0.3527 & 0.0639 & 0.0181 & 0.0061 & -0.0462 & 0.304 \\
\hline No. of fruits / plant & -0.0034 & -0.0154 & 0.0110 & 0.0151 & 0.0212 & 0.0208 & -0.0225 & 0.7934 & -0.0187 & 0.0121 & 0.0040 & 0.1261 & 0.944 \\
\hline Fruit length (cm) & -0.0169 & 0.0785 & 0.0480 & 0.0180 & -0.0063 & 0.0089 & 0.0164 & 0.0751 & -0.1977 & 0.0150 & 0.0050 & 0.1576 & 0.202 \\
\hline Fruit width (cm) & -0.1032 & 0.0813 & 0.0697 & 0.0092 & 0.0103 & -0.0320 & 0.0148 & -0.1544 & 0.0477 & -0.0620 & -0.0190 & -0.0844 & -0.222 \\
\hline $\begin{array}{c}\text { Fruit } \\
\text { circumference }(\mathrm{cm})\end{array}$ & -0.1018 & 0.0796 & 0.0678 & 0.0094 & 0.0094 & -0.0311 & 0.0148 & -0.1537 & 0.0476 & -0.0567 & -0.0208 & -0.0826 & -0.218 \\
\hline Weight of fruit (g) & 0.0294 & 0.0001 & -0.0003 & -0.0066 & 0.0122 & 0.0066 & 0.0055 & 0.2361 & -0.0736 & 0.0123 & 0.0041 & 0.4235 & 0.650 \\
\hline
\end{tabular}


Table.3 Clustering pattern of twenty genotypes of Brinjal

\begin{tabular}{|c|c|c|c|}
\hline S. No. & Cluster & No. of genotypes & Name of genotypes \\
\hline $\mathbf{1}$ & I & 5 & RCMBL 04-04-04, BR 14, AZAD BRINJAL, JB 8 \& \\
PRATIBHA
\end{tabular}

Table.4 Cluster mean of different characters in Brinjal

\begin{tabular}{|c|c|c|c|c|c|c|c|c|c|c|c|}
\hline Cluster & $\begin{array}{c}\text { Plant } \\
\text { height } \\
\text { (cm) }\end{array}$ & $\begin{array}{c}\text { Leaves } \\
\text { per plant }\end{array}$ & $\begin{array}{l}\text { Branches } \\
\text { per plant }\end{array}$ & $\begin{array}{c}\text { Days to } \\
\text { first } \\
\text { flowering }\end{array}$ & $\begin{array}{c}\text { Days to } \\
50 \% \\
\text { flowering }\end{array}$ & $\begin{array}{c}\text { Days to } \\
\text { first } \\
\text { picking }\end{array}$ & $\begin{array}{c}\text { Fruiting } \\
\text { span }\end{array}$ & $\begin{array}{c}\text { Fruits } \\
\text { per } \\
\text { plant }\end{array}$ & $\begin{array}{c}\text { Fruit } \\
\text { length } \\
\text { (cm) }\end{array}$ & $\begin{array}{c}\text { Fruit } \\
\text { weight } \\
\text { (g) }\end{array}$ & $\begin{array}{c}\text { Fruit } \\
\text { yield } \\
\text { per } \\
\text { plant }\end{array}$ \\
\hline I & 76.5 & 36.2 & 10.2 & 40.00 & 60.20 & 62.45 & 52.30 & 12.50 & 12.5 & 5.02 & 2.25 \\
\hline II & 78.2 & 38.2 & 9.60 & 41.60 & 58.80 & 60.15 & 54.50 & 11.60 & 15.00 & 5.00 & 2.15 \\
\hline III & 74.4 & 32.2 & 11.5 & 42.20 & 61.55 & 63.00 & 51.65 & 13.00 & 13.60 & 6.35 & 3.00 \\
\hline IV & 81.5 & 36.2 & 10.8 & 41.60 & 57.50 & 60.65 & 50.20 & 14.20 & 12.55 & 5.80 & 2.50 \\
\hline V & 80.6 & 33.4 & 10.2 & 41.00 & 56.00 & 61.40 & 53.60 & 14.10 & 11.50 & 6.20 & 2.75 \\
\hline
\end{tabular}


The result revealed that magnitude of genotype correlation coefficients were found higher than their II II corresponding phenotypic coefficient of correlation for all traits which indicated inherit association between traits. These findings are close harmony to Naligadhava et al., (2007), shende et al., (2014) and Patel et al., (2015). It further revealed that days to $50 \%$ flowering, days to first picking, fruiting Span, number of fruits per plant, fruit length, fruit weight recorded significant positive association with fruit yield, while it showed non significant association with plant height, number of leaves per plant at 90 DAT, days to first flowering, fruit width and fruit circumference.

Maximum positive direct effects were found on fruit yield by number of fruits per plant followed by leaves per plant at 90 DAT and branches per plant at 90 DAT, where as negative direct effect noticed on fruit yield by fruit length, plant height and days to first flowering which confirmed the findings of Nair and Mehta (2007), Singh et. al (2010) Shende et. al. (2014) and Patel et. al. (2015).

Grouping of genotypes into various clusters revealed that maximum number of genotypes grouped 9 in cluster II, seven in cluster III, nine in cluster I, Three in cluster IV and one in cluster v. Further the grouping of genotypes into various clusters has no relationship of genotypes with their place of origin. These results are in close agreement with results of Sharma and Murya (2004), Singh et al., (2005), Naik (2006) Nair and Mehta (2007). Similarly the cluster mean values for different traits revealed that cluster IV showed maximum mean value for plant height and fruit per plant followed by cluster II for number of leaves per plant, fruit length and cluster III for branches per plant, days to $50 \%$ flowering, fruit weight and fruit yield per plant. The averages inter and intra cluster distance exhibited highest in III followed by cluster IV cluster II and cluster I Neha et al., (2017) and Anil Bhushan et al., (2018).

Overall genotype from cluster III namely BCB- 464, Utkal Tarahi, KS- 331 and DBL24 and from cluster IV, genotypes Aruna, PLR-1 and JB-69 can be used for further breeding work to create maximum diversity in the population can be developed better brinjal commercial variety.

In conclusion the twenty five brinjal genotypes where studied for identification of traits governing fruit yield through correlation and path analysis revealed that days to $50 \%$ flowering fruit span, number of fruits per plant, fruit length, fruit weight and branches per plant found major fruit yield traits. similarly 25 genotypes where grouped in 5 cluster consisting nine cluster II, seven clusters III, nine in cluster I, three in cluster IV and one in plaster $\mathrm{v}$, finally genotypes namely BCB-464, Utkal Tarahi, KS-331, Aruna, PLR-1 and JB- 69 were found superior for further breeding program.

\section{References}

Anonymous. Improved cultivation practice of horticultural crops University of Agriculture science, Dhawa 2012 - 173 -183 .

Mahalanobis, P.C. (1936) on the generalized distance in statistics proceeding of National Academy e off sciences 1936:19: 201 - 208.

Mehta. N. and Sahu, M. (2009). Genetics divergence in Brinjal (Solanum melongena L.). International Journal of plant science 4: 123- 124.

Naik, K.C.K, Genetic variability and divergence studies in Brinjal MSc horti. thesis University agric. science Dharwad 2005.

Rao, C.R. Advanced statistics methods in biometrical research John willey and 
sons. New York 1952- 357-359.

Singh, R.K., Choudhary, B.D. Biometrical methods in quantitative genetic analysis Kalyani publishers New Delhi 1977.

Vadodaria, M.A., a Kulkarni, G.H. Madaviya,
R.B. and dobariya K. L. (2009). Stability for fruit yield and its components traits in Brinjal crop improvement 36 (1) 81-87.

\section{How to cite this article:}

Bhagwan Bamaniya, S. S. Singh and Anita Parmar. 2021. Study of Breeding Potential of Brinjal Genotypes (Solanum melongena L.). Int.J.Curr.Microbiol.App.Sci. 10(01): 3130-3136. doi: https://doi.org/10.20546/ijcmas.2021.1001.364 